\title{
Applications of differential geometry to cartography
}

\author{
Julio Benítez and Néstor Thome \\ Universidad Politécnica de Valencia \\ Departamento de Matemática Aplicada \\ 46071 - Valencia - Spain \\ E-mail: \{jbenitez, njthome\}@mat.upv.es
}

\begin{abstract}
This work introduces an application of differential geometry to cartography. The mathematical aspects of some geographical projections of Earth surface are revealed together with some of its more important properties.
\end{abstract}

\section{Introduction}

An important problem since the discovery of the "spherical" form of the Earth (an inaccurate assumption that will be accepted in this paper for the sake of the studies' needs) is how to compose a reliable map of the surface of the Earth that could be proven useful for navigation. In many cartography texts a scarce mention is made to the mathematics inherent to mathematical development. At the same time, the few texts on differential geometry (or vector calculus) that consider this problem incur in some pedagogical deficiencies: a) the historical origin of the problem is not generally mentioned, b) not always concepts are defined with clear explanation of their origin and application, c) formulas are used with no so much explanation of their obtaining process.

This work intends to find a solution for this void in both scientific disciplines: mathematics and the geography. The content is easily understandable for a first or second year university student (with a certain degree of knowledge on calculus of several variables) and it can be used as support in the study of Euclidean space surfaces. The aim is not a thorough study of terrestrial projections, but an overall view of the difficulties that arise by means of the study of some important projections. The name projection derives from the surface of the Earth being projected in different manners, in a plane, a cylinder or a cone (all surfaces with null Gaussian curvature). The relevance of this work lies in a new approach that we use to present some classical projections (see [2] for reviewing the usual projections). This work aims to show how methods from calculus can also be a valid alternative. The intuitive ideas that a student needs for understanding calculus of several variables are reinforced.

First, let us recall some notation. The partial derivative of a function $f=f(u, v)$ relative to variable $u$ will be denoted by $f_{u}$. All functions will be considered as differentiable as many times as necessary. The usual inner product in $\mathbb{R}^{3}$ of vectors $\mathbf{u}$ and $\mathbf{v}$ will be denoted by $\langle\mathbf{u}, \mathbf{v}\rangle$; while the Euclidean norm of vector $\mathbf{u}$ is denoted by $\|\mathbf{u}\|$. Lastly, $\lambda$ will always denote the geographical longitude; and $\phi$ will denote the geographical latitude. Both angles are measured in radians; thus, $\lambda \in[0,2 \pi[$ and $\phi \in]-\pi / 2, \pi / 2[$. The radius of the Earth will be denoted by $R$. 


\section{Maps and the conformality property}

A map is a piece of paper representing the Earth partially. Mathematically speaking, a map is a subset $D$ of $\mathbb{R}^{2}$, such that given a point $(u, v) \in D$ (in the map), an unique determinate point in Earth can be associated to it. The situation is modelled according to a function $\mathbf{r}$ as follows:

$$
\mathbf{r}: D \rightarrow \text { Earth, } \quad \mathbf{r}=\mathbf{r}(u, v), \quad(u, v) \in D
$$

It is well-known that the surface of a sphere cannot be represented in a plane without a simultaneous distortion in lengths, angles and areas. This statement is a trivial consequence of the Gauss egregium theorem (for example, see [1, p. 234]). Therefore, a map representing the Earth without distortions cannot be proposed. The angles in the map need to be preserved for the sake of practical calculations in navigation. Therefore, if the angle between two curves is measured in the map, then, it should be equal to the angle between the curves on the Earth. This is a fundamental property, since angles point towards the correct course.

What does model (1) require in order to preserve its angles? (This property is known in cartography as conformal). We denote by $E=\left\langle\mathbf{r}_{u}, \mathbf{r}_{u}\right\rangle, F=\left\langle\mathbf{r}_{u}, \mathbf{r}_{v}\right\rangle$ and $G=\left\langle\mathbf{r}_{v}, \mathbf{r}_{v}\right\rangle$.

Since straight lines in the map $u=$ constant, $v=$ constant are perpendicular, if model (1) preserves angles then $F=0$.

On the other hand, for $(a, b) \neq(0,0)$,

$$
\alpha(t)=\left(u_{0}, v_{0}\right)+t(1,0), \quad \beta(t)=\left(u_{0}, v_{0}\right)+t(a, b),
$$

are the parametric equations of two straight lines in the map that intersect when $t=0$, that is to say, in the point $P_{0}=\left(u_{0}, v_{0}\right)$. Evidently, if $\psi$ is the angle formed by these straight lines, then $\cos \psi=a / \sqrt{a^{2}+b^{2}}$. As $\mathbf{r}$ must preserve angles, $\psi$ is the angle formed by $\mathbf{r}(\alpha(t))$ and $\mathbf{r}(\beta(t))$ in Earth, which is nothing more than the angle determinated by their tangent vectors in $\mathbf{r}\left(P_{0}\right)$. Now, it is easy to check that

$$
\left.\frac{\mathrm{d}(\mathbf{r}(\alpha(t))}{\mathrm{d} t}\right|_{t=0}=\mathbf{r}_{u}\left(P_{0}\right) \quad \text { and }\left.\quad \frac{\mathrm{d}(\mathbf{r}(\beta(t))}{\mathrm{d} t}\right|_{t=0}=a \mathbf{r}_{u}\left(P_{0}\right)+b \mathbf{r}_{v}\left(P_{0}\right) .
$$

Since $F=0$, then

$$
\cos \psi=\frac{\left\langle\left.\frac{\mathrm{d}(\mathbf{r}(\alpha(t))}{\mathrm{d} t}\right|_{t=0},\left.\frac{\mathrm{d}(\mathbf{r}(\alpha(t))}{\mathrm{d} t}\right|_{t=0}\right\rangle}{\left\|\left.\frac{\mathrm{d}(\mathbf{r}(\alpha(t))}{\mathrm{d} t}\right|_{t=0}\right\| \cdot\left\|\left.\frac{\mathrm{d}(\mathbf{r}(\alpha(t))}{\mathrm{d} t}\right|_{t=0}\right\|}=\frac{a E}{\sqrt{E} \sqrt{a^{2} E+b^{2} G}} .
$$

Comparing this last equation with $\cos \psi=a / \sqrt{a^{2}+b^{2}}$ and simplifying then $E=G$ is obtained.

Also, obviously, if $E=G$ and $F=0$, then $\mathbf{r}$ preserves angles. Therefore, the following result is obtained:

THEOREM 2.1 Function $\mathbf{r}$ preserves angles if and only if $E=G$ and $F=0$. 


\section{The Mercator projection.}

Let us suppose that the map is rectangular ${ }^{1}$, equally spaced meridians are represented on the map with equally spaced vertical lines (which requires the introduction of a constant $A$ such that $\Delta u=$ $A \Delta \lambda)$, equally spaced parallels are represented on the map with equally spaced horizontal lines (which again requires the introduction of another constant $B$ such that $\Delta v=B \Delta \phi$ ). Therefore, if $H$ is the horizontal width of the map and if $V$ is the vertical width,

$$
\mathbf{r}:[0, H] \times[0, V] \rightarrow \text { Earth }
$$

then, the point on the map with coordinates $(u, v)$ corresponds to the point on the Earth with longitude $\lambda(u)=A u$ and latitude $\phi(v)=B v$ (see figure 1). Then,

$$
\mathbf{r}: D \rightarrow \text { Earth, } \quad \mathbf{r}(u, v)=R(\cos (B v) \cos (A u), \cos (B v) \sin (A u), \sin (B v)),
$$

and functions $E=R^{2} A^{2} \cos ^{2}(B v)$ and $G=R^{2} B^{2}$ are clearly different. So, for theorem 2.1 , this model does not preserve angles.
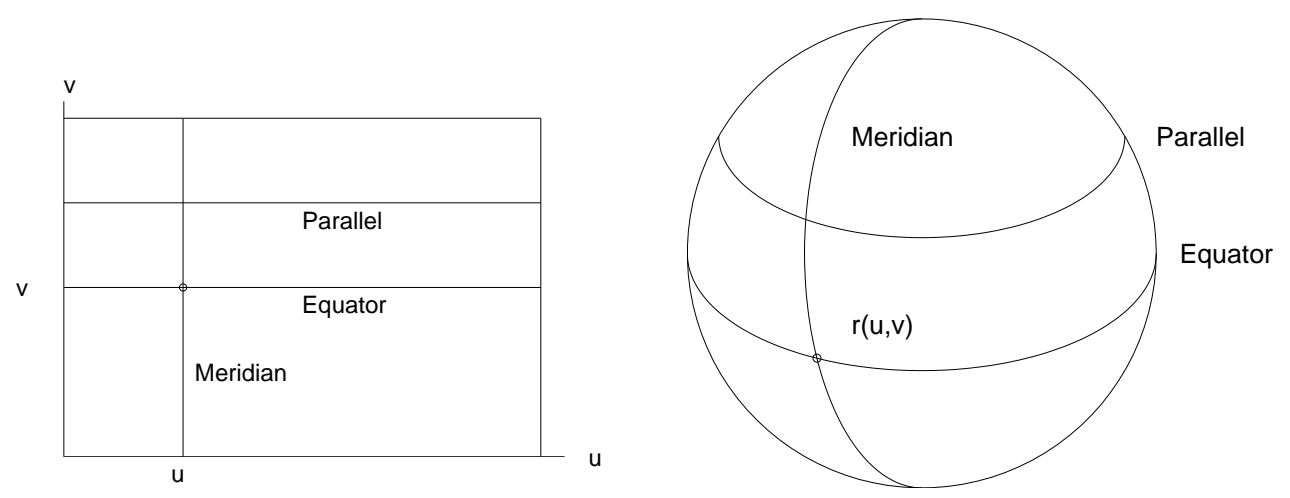

Figure 1: Representation of the Earth on a rectangular map.

It is natural then to modify this model so that it is able to preserve angles. In order to do this, let $\lambda(u)$ be the latitude of point $\mathbf{r}(u, v)$ and $\phi(v)$ its longitude. The same as before, the meridians are represented by means of vertical lines and the parallels with horizontal lines. Then,

$$
\mathbf{r}: D \rightarrow \text { Earth, } \quad \mathbf{r}(u, v)=R(\cos (\phi(v)) \cos (\lambda(u)), \cos (\phi(v)) \sin (\lambda(u)), \sin (\phi(v))) .
$$

It is easy to check that

$$
E=R^{2}\left(\frac{\mathrm{d} \lambda}{\mathrm{d} u}\right)^{2} \cos ^{2}(\phi(v)), \quad F=0, \quad G=R^{2}\left(\frac{\mathrm{d} \phi}{\mathrm{d} v}\right)^{2}
$$

In order for function $\mathbf{r}$ to preserve angles, it must $E=G$ and $F=0$ hold; that is to say, $\cos (\phi(v)) \mathrm{d} \lambda / \mathrm{d} u= \pm \mathrm{d} \phi / \mathrm{d} v$. The negative sign is discarded since both $\lambda$ as $\phi$ are supposed to be

\footnotetext{
${ }^{1}$ Evidently, not all maps are rectangular; but, a simple type of map will be studied.
} 
increasing functions (this assumption will also be applied later on). And so,

$$
\frac{\mathrm{d} \lambda}{\mathrm{d} u}=\frac{1}{\cos \phi} \frac{\mathrm{d} \phi}{\mathrm{d} v} .
$$

Since the first member of (3) only depends on $u$, and the second member of (3) only depends on $v$, both members must be equal to a constant $K$. Now, two trivial differential equations arise that need to be solved:

$$
\frac{\mathrm{d} \lambda}{\mathrm{d} u}=K, \quad \frac{1}{\cos \phi(v)} \frac{\mathrm{d} \phi}{\mathrm{d} v}=K
$$

whose respective solutions are the following:

$$
u=\frac{1}{K} \int \mathrm{d} \lambda=\frac{\lambda}{K}, \quad v=\frac{1}{K} \int \frac{\mathrm{d} \phi}{\cos \phi}=\frac{1}{K} \log \left(\tan \left(\frac{\phi}{2}+\frac{\pi}{4}\right)\right) .
$$

The Mercator projection has just been found. Mercator is the Latinised name of the Flemish cartographer Gerard Kremer, who obtained this projection in 1569 with different methods than those presented in this paper (see for example the paper [4] about the Mercator projection and how Gerard Kremer developed it).

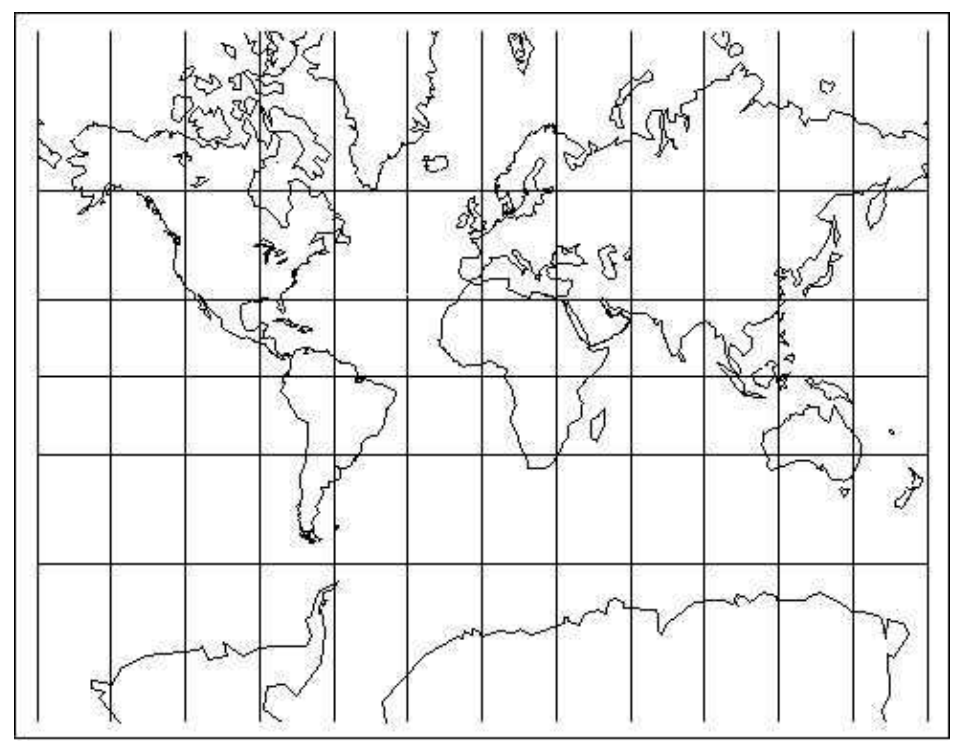

Figure 2: Mercator projection.

The constant $K$ is highly related with the scale of the map. It is easily proven that $1 \mathrm{~cm}$ in the map's equator represents $K R \mathrm{~cm}$ on the surface of the Earth. However, $1 \mathrm{~cm}$ along a parallel of latitude $\phi$, corresponds to $K R \cos \phi \mathrm{cm}$ on the map. The Mercator projection highly distorts distances (and areas as we will see) around the poles. Normally, the Mercator projection is only used when regions are not too close to the poles. 
The Mercator projection presents another important advantage. Loxodromes (constant direction curves on the Earth) are important in navigation. What form will loxodromes have in a Mercator projection?

Since a loxodrome forms a constant angle with the meridians, the representation of a loxodrome on the map forms a constant angle with the verticals. If $\psi$ is this angle, then it is easy to deduce that the equation of the loxodrome (on the map) is the straight line $u=v \tan \psi+C$. This is very important, since the loxodromes are widely used for navigation, and in a Mercator projection these are represented with the simplest curve: the straight line. Also, for (5),

$$
\lambda=\log \left(\tan \left(\frac{\phi}{2}+\frac{\pi}{4}\right)\right) \tan \psi+c
$$

where $c$ is a constant that is found by knowing that the loxodrome passes through a given point.

\subsection{The Mercator projection is not suitable for international politics}

The Mercator projection is suitable for navigating. However, it is not useful in politics neither or in geography teaching. This projection highly distorts areas, as seen in figure 2. As an example, India and Scandinavia seem to be of the same size and in fact, India is over three times larger that Scandinavia. And something similar occurs with Europe and South America; when, South America is almost twice the size of Europe! Let us see the reason for this.

Let $D=\left[u_{1}, u_{2}\right] \times\left[v_{1}, v_{2}\right]$ be a rectangular piece of the Mercator projection. This region, represented on the Earth, is $\mathbf{r}(D)$. Since $E=G$ and $F=0$, then

$$
\operatorname{Area}(\mathbf{r}(D))=\iint_{D} \sqrt{E G-F^{2}} \mathrm{~d} u \mathrm{~d} v=\iint_{D} E \mathrm{~d} u \mathrm{~d} v .
$$

When using equations (2) and (4), we obtain $E=G=R^{2}(\mathrm{~d} \phi / \mathrm{d} v)^{2}=R^{2} K^{2} \cos ^{2} \phi(v)$; then, denoting $\Delta u=u_{2}-u_{1}$,

$$
\operatorname{Area}(\mathbf{r}(D))=\iint_{D} R^{2} K^{2} \cos ^{2} \phi(v) \mathrm{d} u \mathrm{~d} v=R^{2} K^{2} \Delta u \int_{v_{1}}^{v_{2}} \cos ^{2} \phi(v) \mathrm{d} v
$$

This integral will not be computed (although it can be done, since $\cos \phi=1 / \cosh (K v)$ can be obtained from (4)); but it will only be analyzed in a qualitative way. Due to factor $\Delta u$ in (7), the area depends on the increment of longitudes; but not on the longitudes. This indicates that the Mercator projection does not distort distances in the East-West direction.

However, since $\cos ^{2} \phi(v)$ decreases as $\phi$ approaches $\pm \pi / 2$ (the poles) we can conclude that as $D$ moves away from the equator, then $\mathbf{r}(D)$ will have smaller area. This explains why two regions with the same area on the Earth have different sizes on the Mercator projection, since the area closer to the poles will be represented as larger than the other one.

\section{The stereographic projection}

As we have seen, the Mercator projection is not suitable for polar region cartography. For these particular regions stereographic projection (and other methods) are used instead.

Let us consider the Earth with equation $x^{2}+y^{2}+z^{2}=R^{2}$ and oriented in such a way that the North Pole is at $(0,0, R)$. Given $(u, v) \in \mathbb{R}^{2}$, the point $\mathbf{r}(u, v)$ is defined as the intersection 
of the terrestrial surface with the straight line joining the North Pole with point $(u, v,-R)$. The following statement can be proven:

$$
\mathbf{r}(u, v)=\frac{R}{u^{2}+v^{2}+4 R^{2}}\left(4 R u, 4 R v, u^{2}+v^{2}-4 R^{2}\right), \quad(u, v) \in \mathbb{R}^{2},
$$

as well as

$$
E=G=\left(\frac{4 R^{2}}{u^{2}+v^{2}+4 R^{2}}\right)^{2}, \quad F=0,
$$

which indicates that the stereographic projection preserves angles.

Let $D$ be the circle (on the map) with its center in the origin and with radius $\sigma$. It is an exercise (based on a computation of a double integral using polar coordinates and equation (6)), which in the area of $\mathbf{r}(D)$ (on the Earth) is $4 \pi R^{2} \sigma^{2} /\left(\sigma^{2}+4 R^{2}\right)$ [See that if $\sigma \rightarrow \infty$ the area of the Earth is obtained. These types of verifications are very helpful for teaching]. So

$$
\operatorname{Area}(D)-\operatorname{Area}(\mathbf{r}(D))=\pi \sigma^{2}-\frac{4 \pi R^{2} \sigma^{2}}{\sigma^{2}+4 R^{2}}=\frac{\pi \sigma^{4}}{\sigma^{2}+4 R^{2}},
$$

which explains why the stereographic projection faithfully represents areas close to the South Pole; but distorts areas closer to the North Pole.

Next, a more in-depth study of a stereographic projection will be shown. In this projection, meridians are represented by means of semi-straight lines and parallels are represented by means of concentric circumferences. Is this the only projection satisfying both this property and the conformality property? Evidently, using polar coordinates $\rho, \theta$ on the map, instead of Cartesian coordinates $u, v$, is suitable. Which property should $\mathbf{r}=\mathbf{r}(\rho, \theta)$ satisfy in order to preserve angles? The characterization of theorem 2.1 will be used. In order to do this, coefficients $E_{p}=\left\langle\mathbf{r}_{\rho}, \mathbf{r}_{\rho}\right\rangle$, $F_{p}=\left\langle\mathbf{r}_{\rho}, \mathbf{r}_{\theta}\right\rangle$ and $G_{p}=\left\langle\mathbf{r}_{\theta}, \mathbf{r}_{\theta}\right\rangle$, will be associated to aforementioned coefficients $E=\left\langle\mathbf{r}_{u}, \mathbf{r}_{u}\right\rangle$, $F=\left\langle\mathbf{r}_{u}, \mathbf{r}_{v}\right\rangle$ and $G=\left\langle\mathbf{r}_{v}, \mathbf{r}_{v}\right\rangle$. Since $u=\rho \cos \theta, v=\rho \sin \theta$, according to the chain rule,

$$
\mathbf{r}_{\rho}=\mathbf{r}_{u} \cos \theta+\mathbf{r}_{v} \sin \theta, \quad \mathbf{r}_{\theta}=-\rho \mathbf{r}_{u} \sin \theta+\rho \mathbf{r}_{v} \cos \theta .
$$

We shall use matrices in order to simplify the calculations. Equations (9) can be written as

$$
\left[\begin{array}{l}
\mathbf{r}_{\rho} \\
\mathbf{r}_{\theta}
\end{array}\right]=\left[\begin{array}{rr}
\cos \theta & \sin \theta \\
-\rho \sin \theta & \rho \cos \theta
\end{array}\right]\left[\begin{array}{l}
\mathbf{r}_{u} \\
\mathbf{r}_{v}
\end{array}\right] \quad \text { that is } D_{p}=P D,
$$

where $\mathbf{r}_{\rho}, \mathbf{r}_{\theta}, \mathbf{r}_{u}, \mathbf{r}_{v}$ are row vectors. Denoting

$$
\mathcal{G}=\left[\begin{array}{cc}
\left\langle\mathbf{r}_{u}, \mathbf{r}_{u}\right\rangle & \left\langle\mathbf{r}_{u}, \mathbf{r}_{v}\right\rangle \\
\left\langle\mathbf{r}_{v}, \mathbf{r}_{u}\right\rangle & \left\langle\mathbf{r}_{v}, \mathbf{r}_{v}\right\rangle
\end{array}\right], \quad \mathcal{G}_{p}=\left[\begin{array}{cc}
\left\langle\mathbf{r}_{\rho}, \mathbf{r}_{\rho}\right\rangle & \left\langle\mathbf{r}_{\rho}, \mathbf{r}_{\theta}\right\rangle \\
\left\langle\mathbf{r}_{\theta}, \mathbf{r}_{\rho}\right\rangle & \left\langle\mathbf{r}_{\theta}, \mathbf{r}_{\theta}\right\rangle
\end{array}\right],
$$

then

$$
\mathcal{G}_{p}=D_{p} D_{p}^{\mathrm{T}}=(P D)(P D)^{\mathrm{T}}=P D D^{\mathrm{T}} P^{\mathrm{T}}=P \mathcal{G} P^{\mathrm{T}} .
$$

Since $P$ is a nonsingular matrix, it is easy to characterize when $\mathbf{r}(\rho, \theta)$ is conformal.

THEOREM 4.1 The function $\mathbf{r}$ preserves angles if and only if $G_{p}=\rho^{2} E_{p}$ and $F_{p}=0$. 
The proof is as follows:

$$
\mathbf{r} \text { is conformal } \Leftrightarrow E=G, F=0 \Leftrightarrow \mathcal{G}=\mu I \Leftrightarrow \mathcal{S}_{p}=\mu P P^{\mathrm{T}} \Leftrightarrow G_{p}=\rho^{2} E_{p}, F_{p}=0,
$$

where $\mu$ is a real function that in this case coincides with $E$ and $G$.

Let us suppose that $\mathbf{r}=\mathbf{r}(\rho, \theta)$ projects meridians in semi-straight lines starting from the origin and parallel in circumferences centred in the origin. Then

$$
\mathbf{r}(\rho, \theta)=R(\cos (\phi(\rho)) \cos (\lambda(\theta)), \cos (\phi(\rho)) \sin (\lambda(\theta)), \sin (\phi(\rho))) .
$$

The same as in section 3 it is easy to check that

$$
E_{p}=\left(R \frac{\mathrm{d} \phi}{\mathrm{d} \rho}\right)^{2}, \quad F_{p}=0, \quad G_{p}=\left(R \cos \phi \frac{\mathrm{d} \lambda}{\mathrm{d} \theta}\right)^{2} .
$$

Using a similar argument to that seen in section 3 , if $\mathbf{r}$ is conform, there exists a constant $K \in \mathbb{R}$ such that

$$
\frac{\rho}{\cos \phi} \frac{\mathrm{d} \phi}{\mathrm{d} \rho}=K=\frac{\mathrm{d} \lambda}{\mathrm{d} \theta}
$$

and solving both differential equations (including the integration constants denoted here by $A$ and $B)$

$$
\tan \left(\frac{\phi}{2}+\frac{\pi}{4}\right)=A \rho^{K}, \quad \lambda=K \theta+B .
$$

If a complete parallel is navigated on Earth, longitude $\lambda$ has an increment of $2 \pi$. On the map, this journey is represented by means of a circumference centred in the origin, in which case, the increment of $\theta$ is of $2 \pi$ and therefore $K=1$. Obviously, the constant $B$ is associated with the meridian of reference. At the moment, Greenwich meridian (Greenwich is a population located near London) is universally accepted as such. From now on, $B=0$ will be taken. Now, from the first equation of (12), the following can be proven

$$
\cos \phi=\frac{2 A \rho}{A^{2} \rho^{2}+1}, \quad \sin \phi=\frac{A^{2} \rho^{2}-1}{A^{2} \rho^{2}+1} .
$$

Substituting these expressions in (11):

$$
\mathbf{r}(\rho, \theta)=\frac{R}{A^{2} \rho^{2}+1}\left(2 A \rho \cos \theta, 2 A \rho \sin \theta, A^{2} \rho^{2}-1\right) .
$$

What is the meaning of the constant $A$ ? First, if the unit of length is denoted by $\mathrm{L}$; since $\mathbf{r}$ is a position and $R, \rho$ are lengths, then the units of $A$ should be $\mathrm{L}^{-1}$. Second, the stereographic projection should be a particular case of (13). Expression (8) in polar coordinates is

$$
\mathbf{r}(\rho, \theta)=\frac{R}{\rho^{2}+4 R^{2}}\left(4 R \rho \cos \theta, 4 R \rho \sin \theta, \rho^{2}-4 R^{2}\right) .
$$

In order for expression (13) to be similar to (14), it must be divided and multiplied by $A^{2}$ in (13); and it is denoted $d=1 / A$, obtaining

$$
\mathbf{r}(\rho, \theta)=\frac{R}{\rho^{2}+d^{2}}\left(2 d \rho \cos \theta, 2 d \rho \sin \theta, \rho^{2}-d^{2}\right) .
$$


Now, it is evident that the stereographic projection is a particular case of (15): it is enough to take $d=2 R$. Curiously, $2 R$ is the distance between the North Pole and the plane where the Earth is projected. Is this by chance? If $r$ is the straight line that joins the North Pole with polar coordinate point $(\rho, \theta)$ (which is located in the horizontal projection plane located $d$ away from the North Pole), then the intersection of the sphere with straight line $r$ is $\mathbf{r}(\rho, \theta)$, which explains the geometric meaning of the constant $d$.

Also, the scale of the map is relationed with this constant $d$, as illustrated in figure 3 , which is the flat equivalent of the stereographic projection.

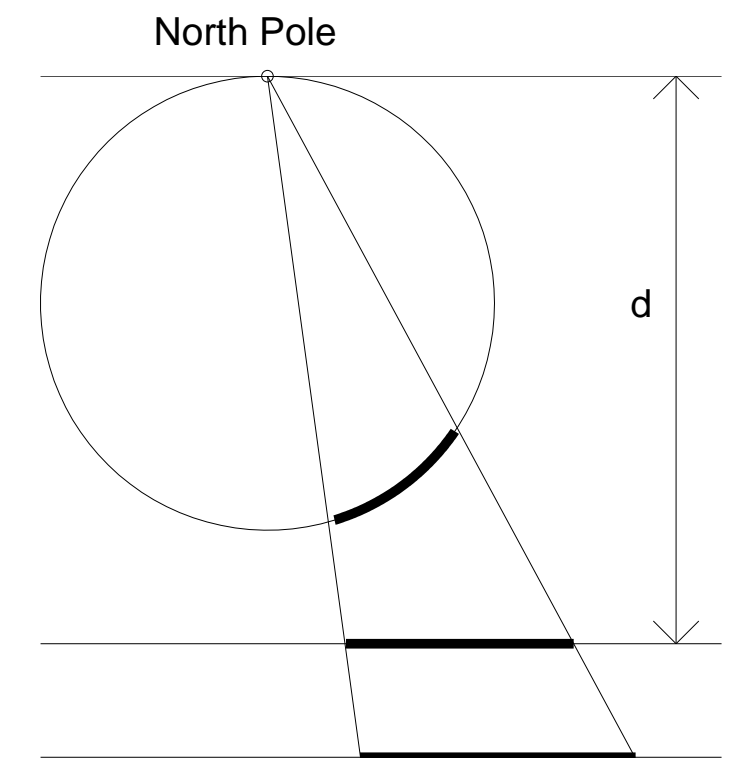

Figure 3: Stereographic projection.

\section{The problem of the areas}

We have just observed how both the stereographic and the Mercator projection do not preserve areas. What condition would be necessary and sufficient so that $\mathbf{r}$ preserves areas?, that is, there exists a constant $K \in \mathbb{R}$ such that $K \cdot \operatorname{Area}(R)=\operatorname{Area}(\mathbf{r}(R))$ for all $R \subset D$. The constant $K$ represents the scale of the map.

Let $\left(u_{0}, v_{0}\right) \in D$ be an arbitrary point of the map, and let $R$ be a region of the map containing this point. Then, according to the theorem of the mean of double integrals, there exists $\left(u_{R}, v_{R}\right) \in$ $R$ such that

$$
K \cdot \operatorname{Area}(R)=\operatorname{Area}(\mathbf{r}(R))=\iint_{R} \sqrt{E G-F^{2}} \mathrm{~d} u \mathrm{~d} v=\left.\operatorname{Area}(R) \cdot \sqrt{E G-F^{2}}\right|_{\left(u_{R}, v_{R}\right)},
$$

that is $K=\left.\sqrt{E G-F^{2}}\right|_{\left(u_{R}, v_{R}\right)}$. Recall that $K$ does not depend on $R$. And making $R \rightarrow\left(u_{0}, v_{0}\right)$, 
then $\left(u_{R}, v_{R}\right) \rightarrow\left(u_{0}, v_{0}\right)$. Hence $\sqrt{E G-F^{2}}$ evaluated in $\left(u_{0}, v_{0}\right)$ equals $K$. Since $\left(u_{0}, v_{0}\right)$ is arbitrary, then $\sqrt{E G-F^{2}}$ is constant (and equals $K$ ). This result can be summarized in the following theorem.

THEOREM 5.1 The function $\mathbf{r}$ preserves areas if and only if $\sqrt{E G-F^{2}}$ is a (positive) constant.

The necessary condition has been shown and the sufficient condition is evident.

\subsection{The Lambert Projection}

As an application of theorem 5.1, we shall find a projection that is able to preserve areas from a model similar to the Mercator model. Let us suppose that the projection

$$
\mathbf{r}: D \rightarrow \text { Earth, } \quad \mathbf{r}(u, v)=R(\cos (\phi(v)) \cos (\lambda(u)), \cos (\phi(v)) \sin (\lambda(u)), \sin (\phi(v)))
$$

preserves areas. Then (2) is satisfied as in the Mercator property. If $\mathbf{r}$ preserves areas, then $\sqrt{E G-F^{2}}$ must be constant; and therefore, there exists $K$ such that

$$
K=\sqrt{E G-F^{2}}=R^{2} \frac{\mathrm{d} \lambda}{\mathrm{d} u} \frac{\mathrm{d} \phi}{\mathrm{d} v} \cos \phi \quad \Longrightarrow \quad \frac{K}{R^{2}} \frac{\mathrm{d} u}{\mathrm{~d} \lambda}=\frac{\mathrm{d} \phi}{\mathrm{d} v} \cos \phi
$$

As the left member only depends on $u$ and the right member only depends on $v$; so there exists a constant $L$ such that

$$
\frac{K}{R^{2}} \frac{\mathrm{d} u}{\mathrm{~d} \lambda}=\frac{\mathrm{d} \phi}{\mathrm{d} v} \cos \phi=L .
$$

Two trivial differential equations arise that need to be solved and whose solutions are (omitting the integration constants)

$$
u=\frac{L R^{2}}{K} \lambda, \quad v=\frac{1}{L} \sin \phi .
$$

The Lambert projection has just been obtained (except for a small modification that will be described next). Why are there two constants $K$ and $L$, when there is only one in the Mercator projection? Due to the fact that if a transformation preserves areas, it is possible distances are increased in an axis and decreased in another, eventhough the areas are preserved (for example, think about the linear transformation of $\mathbb{R}^{2} \rightarrow \mathbb{R}^{2}$ given by $T(x, y)=(\alpha x, y / \alpha)$, where $\left.\alpha \in \mathbb{R} \backslash\{0\}\right)$. A constant will be chosen so that this distortion of lengths is equal for the two axes. This projection preserves areas; but not angles, since

$$
E=\frac{K^{2} \cos ^{2} \phi}{L^{2} R^{2}}, \quad G=\frac{R^{2} L^{2}}{\cos ^{2} \phi}, \quad E \neq G .
$$

Near the equator, the latitude $\phi$ is approximately equal to 0 , and therefore $E \simeq K^{2} /\left(L^{2} R^{2}\right)$ and $G \simeq R^{2} L^{2}$. If near the equator this model (approximately) preserves angles, then $E \simeq G$, (remember that always $F=0$ ). Therefore it is natural to choose for $L$ the value $\sqrt{K} / R$. And so (16) becomes

$$
u=\frac{R}{\sqrt{K}} \lambda \quad \text { and } \quad v=\frac{R}{\sqrt{K}} \sin \phi,
$$

which is the Lambert projection. 


\subsection{The problem of the areas in polar coordinates}

In this subsection we shall find a necessary and sufficient condition so that $\mathbf{r}=\mathbf{r}(\rho, \theta)$ preserves areas. We must consider the condition found at the beginning of section 5 in terms of coefficients $E_{p}, F_{p}, G_{p}$ (defined in section 4 ). But looking for this relationship is an arduous task if matrices are not used. Observe that

$$
E G-F^{2}=\operatorname{det} \mathcal{G}, \quad E_{p} G_{p}-F_{p}^{2}=\operatorname{det} \mathcal{G}_{p}
$$

and because of the relationship (10) among $\mathcal{G}$ and $\mathcal{S}_{p}$, we get

$$
\operatorname{det} \mathcal{G}_{p}=\operatorname{det}\left(P \mathcal{G} P^{\mathrm{T}}\right)=(\operatorname{det} P)^{2} \operatorname{det} \mathcal{G}=\rho^{2} \operatorname{det} \mathcal{G} \text {. }
$$

Now the following theorem is evident.

THEOREM 5.2 The function $\mathbf{r}=\mathbf{r}(\rho, \theta)$ preserves areas if and only if there exists a constant $K>0$ such that $\sqrt{E_{p} G_{p}-F_{p}^{2}}=\rho K$.

Following a similar argument to those considered before, the study of a projection of the type (11) that preserves areas is possible.

\section{Conclusions}

Obviously, not all the existing projections have been studied. The different types of existing projections can be seen in literature on geodesy and cartography [2] has been used for the elaboration of this work). In geodesical studies, geometrical methods are applied to analyse the projections. However, we showed that analytic methods can also be a valid alternative. Observe that a minimum knowledge on calculus of several variables has been the only tool necessary that can be found in [3]. In addition, several types of projections have been naturally found.

Acknowledgements: We would like to thank the ACLE for their help in this paper.

\section{References}

[1] DO CARMO, M. P., 1976, Differential Geometry of Curves and Surfaces. (Prentice-Hall, London)

[2] LAUF, G. B., 1983, Geodesy and Map Projections. (TAEF Publications Unit, Collingwood).

[3] MARSDEN, J. E.; TROMBA, A. J., 1996, Vector Calculus. (W. H. Freeman and Company, New York).

[4] SACHS, J. M., 1987, Mathematics Magazine, vol. 60, no. 3, 151-158. 